\title{
Perceptions of Who is in the Healthcare Team? A Content Analysis of Social Media Posts during COVID-19 Pandemic
}

\section{Alla El-Awaisi ${ }^{1}$, Veronica O'Carroll², Somaya Koraysh¹, Sarra Koummich¹, Marion Huber ${ }^{3}$ \\ ${ }^{1}$ College of Pharmacy, QU Health, Qatar University, Doha, Qatar; ${ }^{2}$ School of Medicine, University of St Andrews, St \\ Andrews, UK; ${ }^{3 Z}$ urich University of Applied Sciences, Switzerland}

\section{Background}

- The COVID-19 pandemic has highlighted that along with physicians and nurses, other professional staff have significant patientfacing roles

- Social media platforms are seen as an instant method to communicate with the public and to provide better understanding of their perception about the roles of healthcare professionals

- As literature related to public perceptions of interprofessional teams is limited, social media content analysis is a potential tool to study public perception of interprofessional teams

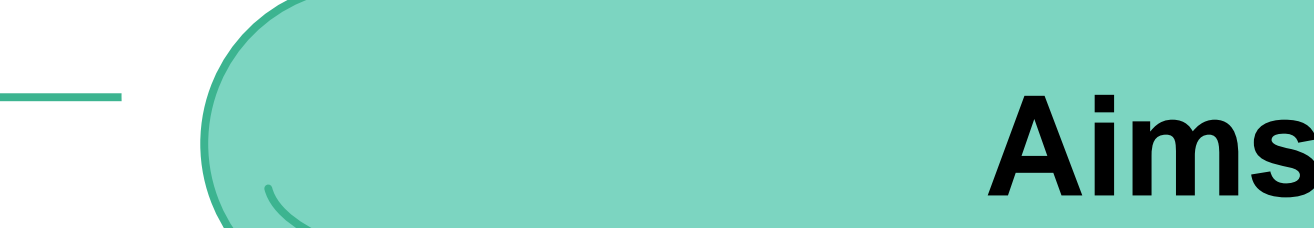

- The aim of this study was to use social listening technique to explore unfiltered public perceptions of the professionals involved in healthcare teams during the COVID-19 pandemic, in a naturalistic, online setting, and to elaborate on the emotional reactions in response to an online social media post

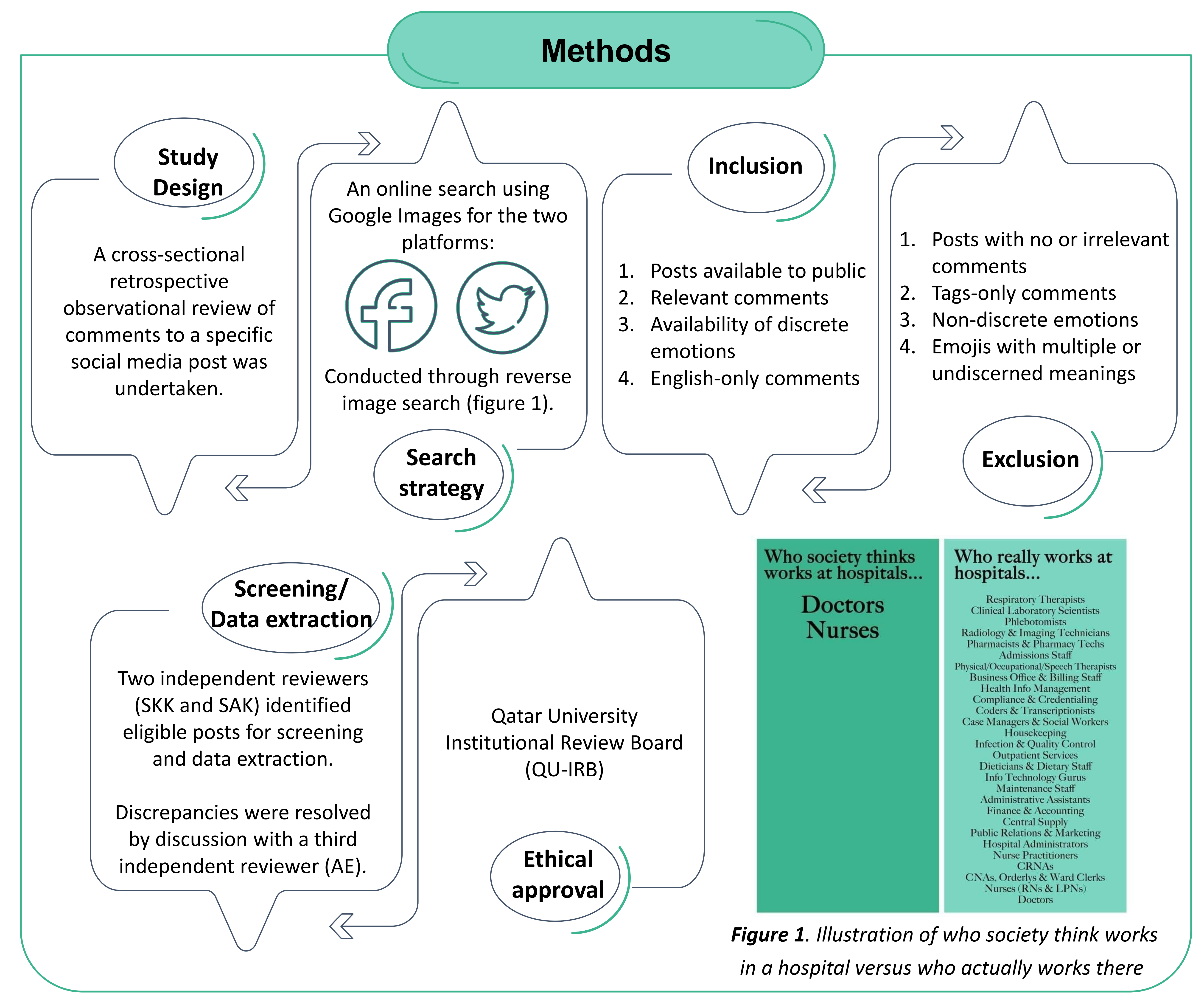

Results

Discrete Emotions Evoked By The Post

Key Highlights Inferred From the Comments
Figure 2. PRISMA chart for the comments' collection

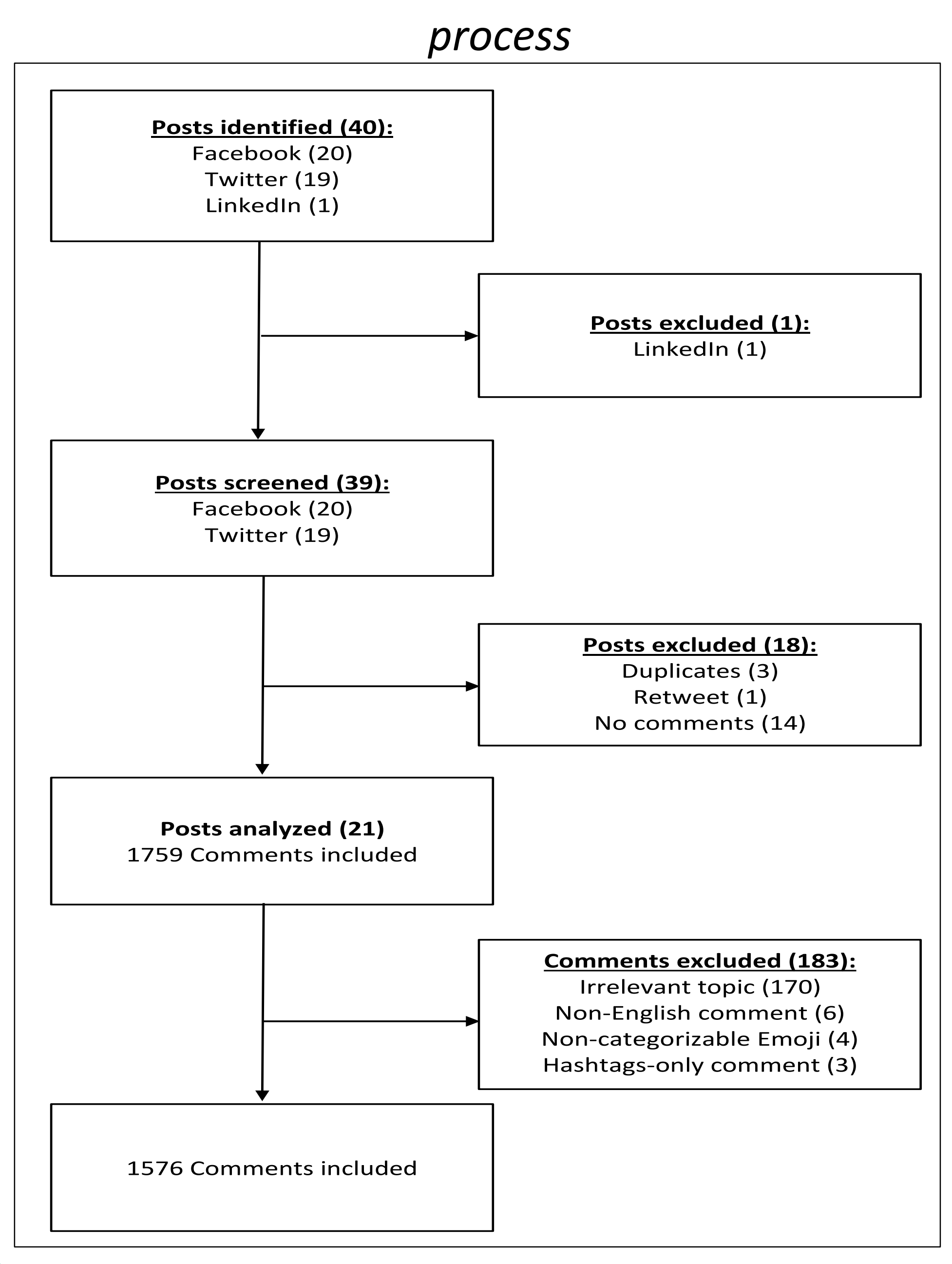

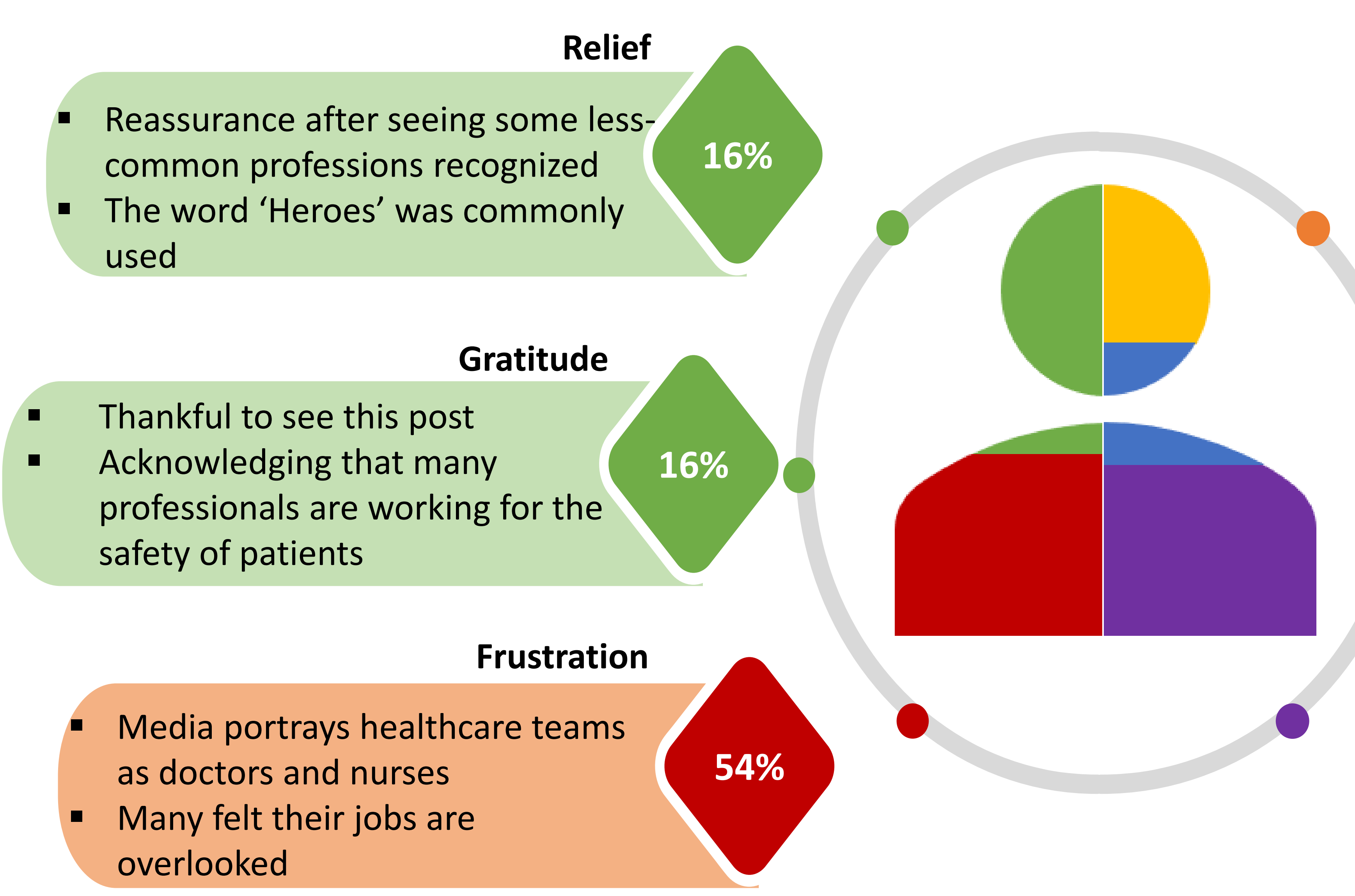

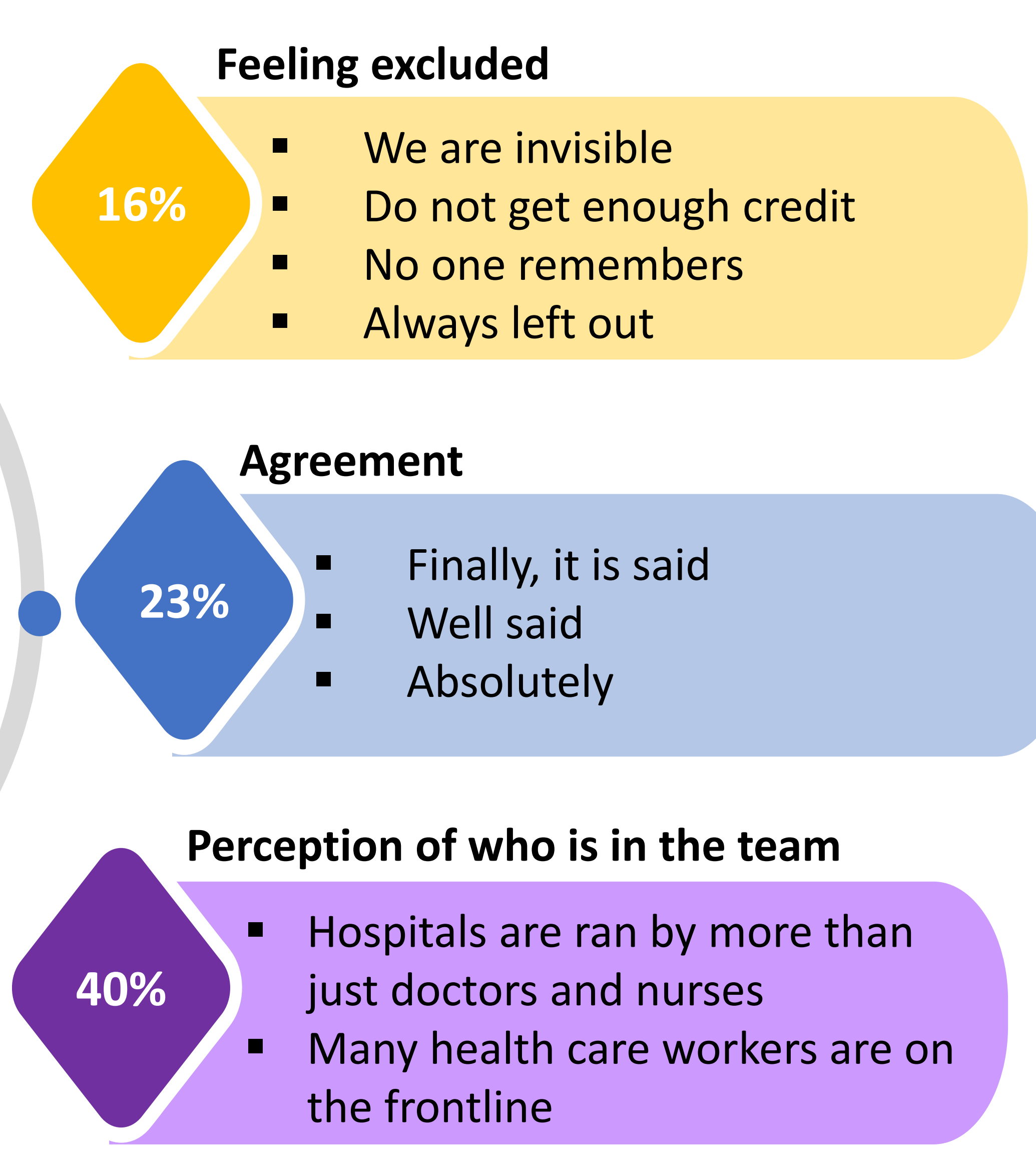

\section{Conclusion}

- The current COVID-19 pandemic and the media attention should be taken as an opportunity by the interprofessional community to work together to combat negative media stereotypes

- It is important to consider how to continue to use social media positively to raise awareness about the interprofessional team

- Further research is needed to increase understanding of public perceptions of interprofessional practice

\section{Limitations}

- A sampling frame could not be used to select the target population

- It was difficult to determine whether the sample were healthcare professionals, patients, or a member of the public

- No inferential statistics were conducted

- These posts may not be entirely representative of the emotions of the wider population as it relied only on publicly available data

\section{Reference}

This study has recently been published:

El-Awaisi A, O'Carroll V, Koraysh $S$, Koummich $S$, Huber $M$ Perceptions of who is in the healthcare team? A content analysis of social media posts during COVID-19 pandemic. Journal of Interprofessional Care. 2020;23:1-1.

DOI: https://doi.org/10.1080/13561820.2020.1819779

.

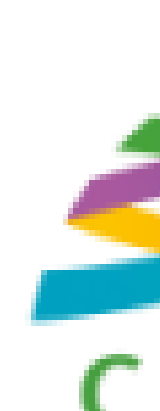

$C A$ I P E

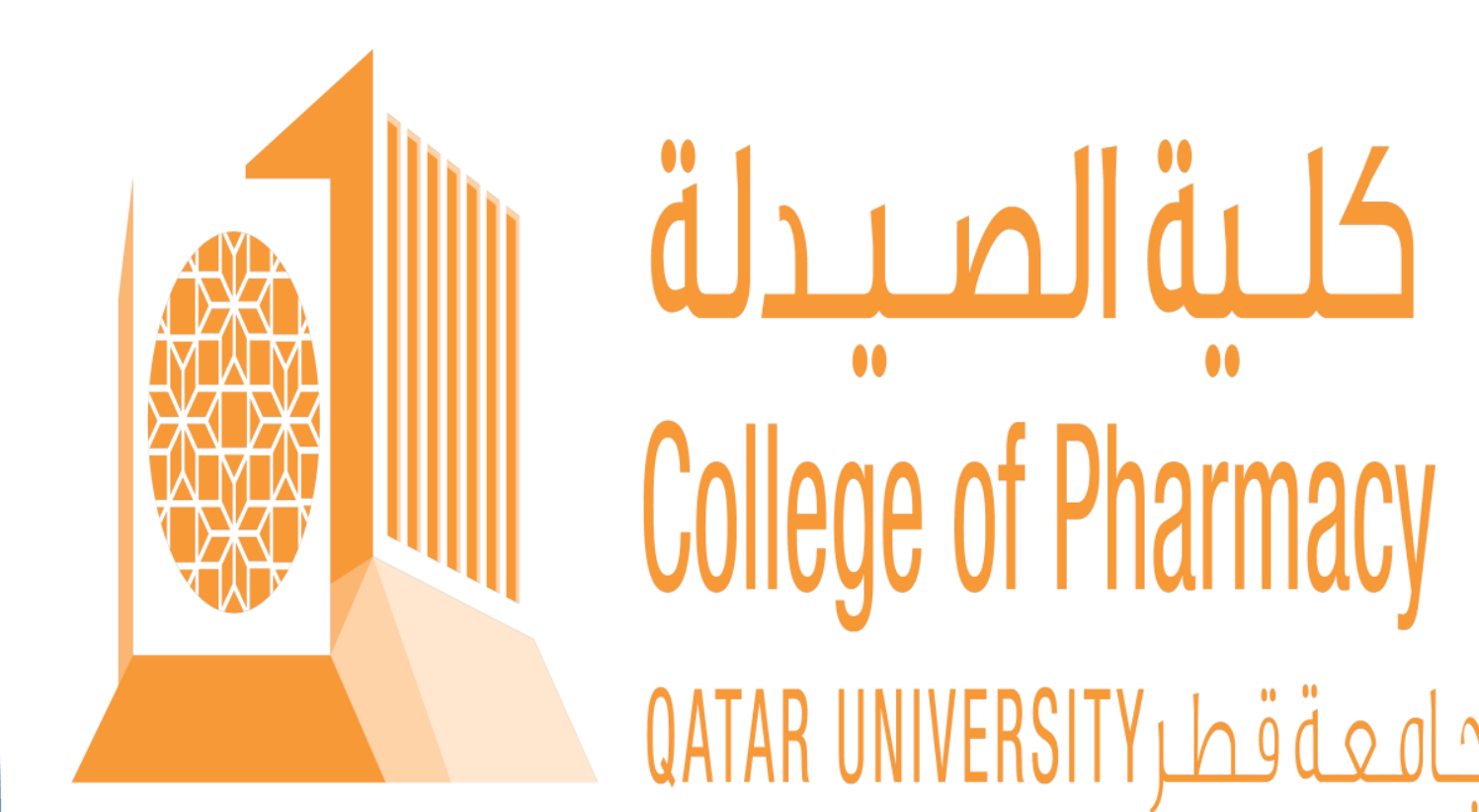

